\title{
Design and Implementation of High-Speed Real-Time Data Acquisition and Processing System based on FPGA
}

\author{
Guojuan Zhou \\ Beijing Institute Of Economics And \\ Management,Beijing,100102 \\ Fuhua Yu \\ Beijing Institute Of Economics And \\ Management,Beijing,100102
}

\begin{abstract}
In this paper, we conduct research on the design and the implementation of the high-speed real-time data acquisition and processing system based on the FPGA. Input signal data acquisition system are most come from the sensor output signal, different types of basic sensors, the signal characteristics are different, each channel signal amplitude and the frequency range is very different, high precision, large dynamic range of $A / D$ transformation chip to make design more can meet the needs of measurement, especially for wide-band weak signal acquisition is especially necessary. In our paper, we propose the data acquisition and processing system based on the FPGA with the basic implementation that serves as the foundation for our further research.
\end{abstract}

Keywords- FPGA, High-Speed, Real-Time, Data Acquisition, Implementation, Processing.

\section{Introduction}

Data acquisition system is the bridge of computer contact with the outside world, is the important way of obtaining information. Data mining technology is an important part of information science, that has been widely used various fields of national economy and national defense construction, and with the development of general science and technology, especially the development of computer technology and popularization, the data mining technology will have wide development prospects.

\author{
Guocan Xiong \\ Beijing Institute Of Economics And \\ Management,Beijing,100102 \\ Wen'E Sun \\ Beijing Institute Of Economics And \\ Management,Beijing,100102
}

The traditional data acquisition system, usually adopts single-chip microcomputer or DSP as main control module, ADC, storage, and other peripheral circuit control work. Along with the speed of data acquisition performance requirements higher, the disadvantages of traditional acquisition system is more and more obvious. MCU clock frequency is low and need software to realize data acquisition, the acquisition speed and efficiency is reduced, in addition the software running time also accounts for the large proportion in the whole sampling time and FPGA has the advantage of microcontroller is unmatched. The FPGA clock frequency is high, the internal time delay is small, all to be finished by the hardware control logic, speed, high efficiency. On the basis of the technology, in order to meet the requirements of data acquisition for speed that are introduced in this paper, a kind of based on FPGA, using VHDL hardware description language design to realize high speed data acquisition system [1].

Big data from birth to now has been a more abstract concepts, if only from the literal meaning interpretation, is the meaning of relatively large amount of data, but this cannot be explained big data in various areas, various disciplines of the differences and the relations. For large data have been no specific definitions of a unified. On Wikipedia, big data refers to the amount of data involved too huge to manually, within a reasonable period of time to capture, 
management, processing, information has become the human can read. The characteristics of the data could be summarized as follows. (1) The diversity of data types is various. In the past, the data type of a single, usually only one or a few, and the data is given priority to with structured data. However, there are many different kinds of data in a large data, and give priority to with unstructured data and semi-structured data. (2) Data authenticity. IBM think big data must have real. Only real data has a reference value. In today's rapid development of informationization process, to requirement of data integrity should be strictly. Any unreasonable, false data will lead to the decision or program error that affects the development of social economy and the life. (3) Data input and output speed quickly. A lot of online or the real-time data processing requirements have been in the era of big data is very important.
VHDL has nothing to do with the specific hardware circuit and has nothing to do with the design platform features, and has good circuit behavior description and the ability of system description, and as easy to read in the language and the hierarchical, structured design, showed a strong vitality and the potential applications. Design method of VHDL, therefore, is to support a variety of patterns, such as top-down and bottom-up or hybrid approach, and in the face of today's many electronic product life cycle shorten, need many times to design into the latest technology in such aspects as changing the process and shows good adaptability. Electronic systems using VHDL design of a big advantage is that the designer can focus on its function, without the need for does not affect the function of the factors related to the process cost too much time and energy. For visualized demonstration, we show the sample time data acquisition and processing system as thr figure one [2-4].

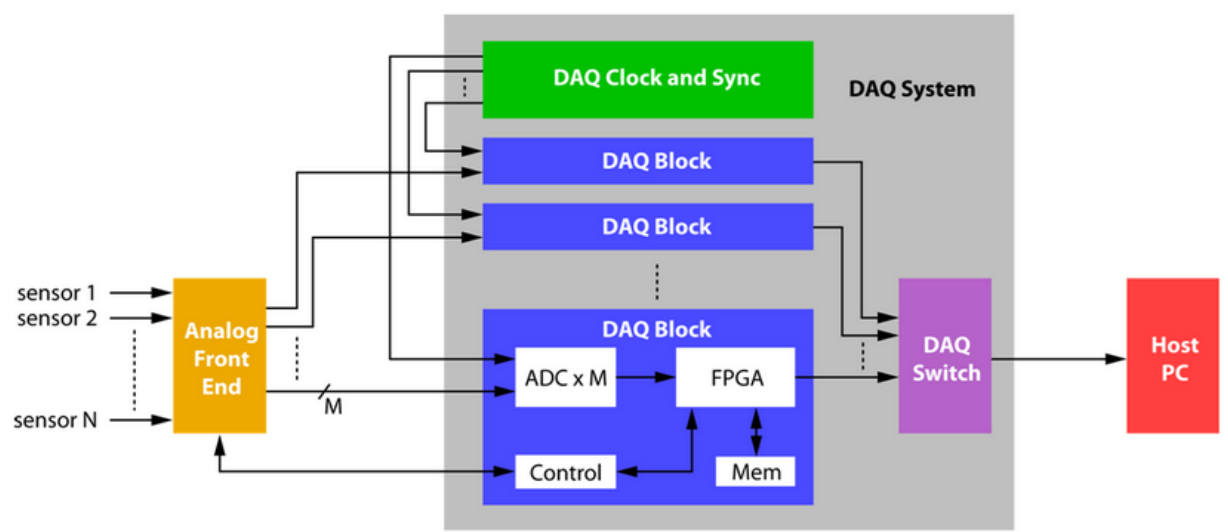

Figure 1. The Sample Time Data Acquisition and Processing System

In this paper, we conduct research on the design and implementation of high-speed real-time data acquisition and processing system based on the FPGA. With the development of general science and technology, data acquisition system has been more and more widely used. For its various technical indicators, higher and higher demands are proposed especially the precision and sampling rate users and designers are more important issues of mutual interest. In the following sub-sections, we will then discuss the corresponding techniques in detail with analysis.

\section{The Proposed Methodology and Perspective}

The Big Data and Cloud. An increasingly large amount of data of the diversity of the application, an increasing number of user requirements, mass data storage is becoming more and more important. Traditional file system already cannot meet the requirement of the massive amounts of 
data storage, in order to meet these new requirements as the distributed file system applied widely attention. How to realize the mass data storage in cloud computing environment becomes an important research topic. With the high-speed development of science and technology level, big data technology is increasingly important, at present the object in the study of data mainly embodies the following four aspects.

- At present, in the practice of big data applications, the object of the application include: search data management, data analysis and general data integration. Among them in the large Internet database and new data storage model and integrated system for research is data management.

- A study on large data in use of basic theory, there are still many problems not fully resolved. At the same time to evaluate data quality and computation efficiency of the process, not a unified evaluating standard, thus lead to low efficiency of the evaluation work of the technical personnel appeared problems [5].

- In the research on the key techniques for large data, the most important problem is that big data format conversion, data transfer and processing problems, but because of the large data has the characteristics of heterogeneous and heterogeneity so try to improve the basic efficiency of the large data format conversion, such ability can effectively applied technology of big data.

- In the study of data security, the most important security problem is the large data privacy and data quality that may influence the performance of the data processing systems.

Cloud computing technology matures, offers these diverse data storage and computing platform. At the same time, such as data mining and artificial intelligence technology for big data era of basic resources, the rapid development of the large data further information openness. Huge amounts of the data storage model is through the number of storage nodes constitute a very large capacity storage system, through a large number of the storage nodes work in parallel to obtain the higher disk access throughput rate, improve the system throughput by system cache to reduce the disk access, through multiple storage nodes fault tolerant improve data reliability, so as to realize the ideal mass storage system of the characteristics of large capacity, high reliability and high performance.

The Data Processing System. Big data consistency problem of distributed systems: consistency problem is mainly in order to guarantee the correctness of the distributed systems for example, in the use of multiple copies to ensure the reliability of the data system, how to ensure that each copy of the data content is exactly the same consistency issues. In order to guarantee system of the high reliability, real-time measurement and the control data processing system adopts double net double cluster hot standby redundancy design technology, consists of three core layer switches, control nodes of cluster management, computing nodes pool, database management and distribution of nodes and the system console that could be reflected from the following figure two.

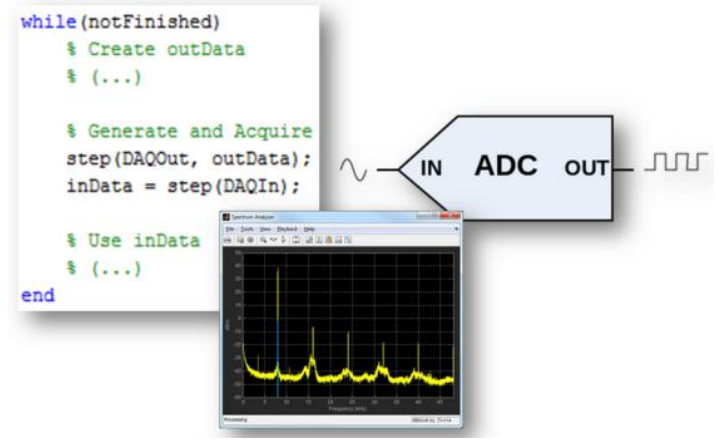

Figure 2. The Real-Time Data Acquisition and Processing Paradigm

Scalability problem is actually a performance problem. For example, for a distributed file 
system, the entire file system running in the node number as many as thousands of the distributed cluster. The basic requirement is to ensure the core performance of balance all machine's disk utilization, network bandwidth utilization and reduce the access to the generation of hot spots with listed suggestions.

- Because the quantity of system, undertake the task of basic constant and computational load, according to the particle size of primary task based on the above method, the dynamic load balancing strategy based on task allocation table, design the real-time processing of data load balance algorithm. When a compute node failure or overload in the real-time cluster system, cluster system monitoring software can detect the abnormal in cluster system within the given time node, and pass this message to the cluster, load balancing software.

- Granularity partition cluster system is a basic issue in cluster node task allocation and task scheduling, moderate granularity partition can improve the efficiency of the task scheduling between nodes, reduce traffic [6-7].

- In order to improve the usability of system, reasonable disposal of abnormal situation, need to establish mechanism of the human-computer interaction management. Through management control node monitoring the load and task migration situation of computing nodes, to found the task of frequent migration, node overload or light load anomalies such as intervention.

The FPGA and Data Analysis Features. The FPGA is in PAL, GAL and EPLD programmable device developed on the basis of further. The FPGA clock frequency is high, the internal time delay is small, fast, all done by the hardware control logic as itself set sampling control, processing, caching, transmission control, communication within a single chip, flexible configuration, short development cycle and programming system is simple which has high integration, small volume, the low power consumption, the I/O port, the system programming, etc. In the high speed multi-channel data acquisition system, the FPGA can overcome the deficiency of the MCU or the DSP and meet the requirements of system for real-time and synchronization. With the rapid development of electronic design automation technology, the use of VHDL or Verilog HDL hardware description language to realize programmable logic devices such as functional design has become a development direction, in most of the digital circuit logic design using VHDL or Verilog HDL as they are by far most popular hardware description language. Therefore, when high speed optical fiber data transmission interface card based on FPGA using VHDL hardware description language to design the code in order to realize high speed serial data transmission as shown in the figure 3 .

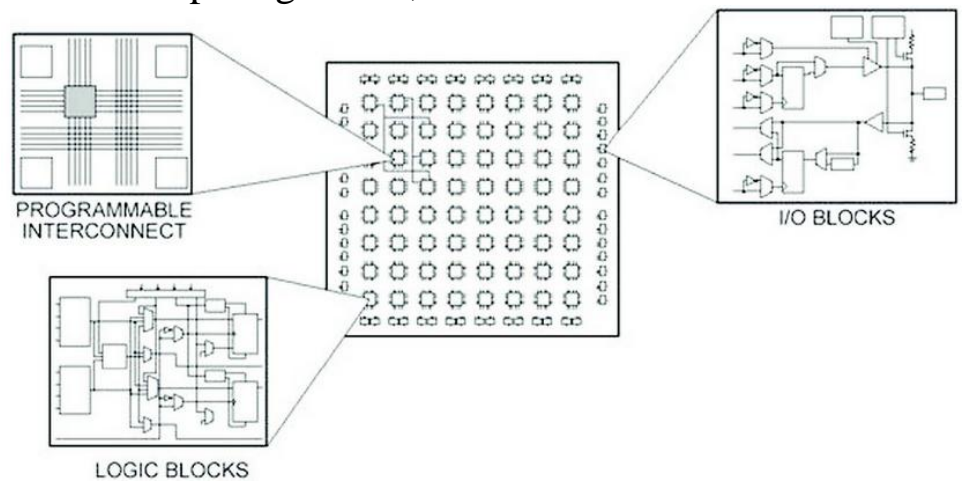

Figure 3. The General Pattern of the FPGA 
The FPGA Data Acquisition. MCU clock frequency is low and use software to realize the data acquisition, acquisition speed and efficiency is reduced, the software running time in make up a large proportion of the whole sampling time and FPGA has the advantage of microcontroller is unmatched. The FPGA clock frequency is high, the internal time delay is small, all to be finished by the hardware control logic, speed, high efficiency. Adaptive amplifier circuit design system based on FPGA including signal filtering, the content of small signal amplifier, signal acquisition and data processing. Input of small signal after filtering circuit into programmable amplifier, programmable amplifier based on FPGA signal to control basic signal magnification, amplifier output signal is sent to $\mathrm{A} / \mathrm{D}$ converter, all the way all the way into the voltage comparator, voltage comparator has A reference voltage, resulting from the amplifier output voltage and reference voltage is the signal to the FPGA, FPGA by calculating the signal after A/D conversion and the signal voltage comparator, the amplifier amplification can be concluded that the control program [8].

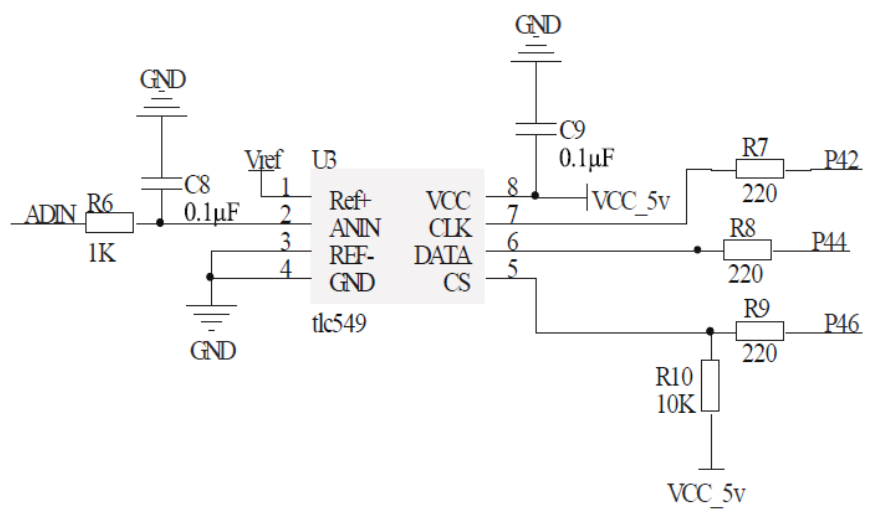

Figure 4. The Data Sampling circuit

As shown in the figure 4, we demonstrate the data sampling circuit. Suitable for complete single channel eight conversion, which fit in with the requirement is not high in speed, consisting of a data acquisition system. Eight bits chips can be easily used with serial interface controller connected with peripheral. According to the eight bits strict timing, it in the completion of $\mathrm{A} / \mathrm{D}$ conversion, the serial output of A0-A7 binary data can be controlled by sequence, and the serial output to the string into and out of the shift register. This experiment complete high-speed, multi-channel data acquisition system of the FPGA design. Using EDA tools and languages to design FPGA, and the system simulation and verification in the EDA software. By the characteristics of FPGA online programming, can according to the specific site conditions, modify the FPGA's internal configuration and further increase the flexibility of the system used, so the system is a kind of ideal high-speed data acquisition scheme.

\section{Conclusion}

In this paper, we conduct research on the design and implementation of the high-speed real-time data acquisition and general processing system based on the FPGA. This paper complete high-speed, multi-channel data acquisition system of the FPGA design, the system has the advantages of simple circuit, small volume. Use EDA tools and languages on the FPGA design, simulation and verification, it is easy to design modification and optimization, greatly shorten the product development design cycle. By FPGA has the characteristics of online programming can be based on the scene of the specific 
circumstances, to modify the internal logic of the FPGA configuration, to further increase the flexibility of system application, achieved satisfactory results by testing the design, is a kind of ideal multi-channel and high-speed data collection scheme. In the future, we will conduct the experimental verification to enhance the current research result.

\section{References}

[1] Prakash, Amol, et al. "Hybrid data acquisition and processing strategies with increased throughput and selectivity: pSMART analysis for global qualitative and quantitative analysis." Journal of proteome research 13.12 (2014): 5415-5430.

[2] Sotiropoulos, Stamatios N., et al. "Advances in diffusion MRI acquisition and processing in the Human Connectome Project." Neuroimage 80 (2013): 125-143.

[3] Desai, S., et al. "The Blanco Cosmology Survey: Data Acquisition, Processing, Calibration, Quality Diagnostics, and Data Release." The Astrophysical Journal 757.1 (2012): 83.

[4] Fritz, Andreas, et al. "Reliable noise and vibration data acquisition and processing for automotive applications." Instrumentation and Measurement Technology Conference (I2MTC) Proceedings, 2014 IEEE International. IEEE, 2014.

[5] Chiu, Shih-Wen, et al. "A signal acquisition and processing chip with built-in cluster for chemiresistive gas sensor array." New Circuits and Systems Conference (NEWCAS), 2014 IEEE 12th International. IEEE, 2014.

[6] Frank, M., et al. "The LHCb Data Acquisition and High Level Trigger Processing Architecture." Journal of Physics: Conference Series. Vol. 664. No. 8. IOP Publishing, 2015.

[7] Tellaeche, A., and R. Arana. "Rapid Data Acquisition System for Complex Algorithm Testing in Plastic Molding Industry." Proceedings of World Academy of Science, Engineering and Technology. No. 79. World Academy of Science, Engineering and Technology (WASET), 2013.

[8] Stumpf, Felix, et al. "Erosion Modeling in Central China-Soil Data Acquisition by Conditioned Latin Hypercube Sampling and Incorporation of Legacy Data." EGU General Assembly Conference Abstracts. Vol. 15. 2013. 